\title{
Characteristics and clinical value of 3D MR imaging in the diagnosis of pulmonary embolism
}

\author{
JIASHOU HU ${ }^{1 *}$, ZHONGWEI LI ${ }^{2 *}$, YANYAN QU ${ }^{3}$, JINFENG SUN ${ }^{4}$, \\ GUOWEI ZHANG $^{2}$ and GUANGHUI ZHANG ${ }^{5}$
}

\author{
${ }^{1}$ Department of Medical Imaging, The People's Liberation Army 107th Hospital, Yantai, Shandong 264002; \\ ${ }^{2}$ CT Room and ${ }^{3}$ Department of Endocrinology, Yantaishan Hospital, Yantai, Shandong 264001; \\ ${ }^{4}$ Cancer Diagnosis and Treatment Center, The People's Liberation Army 107th Hospital, Yantai, Shandong 264002; \\ ${ }^{5}$ Department of Medical Imaging, Yantaishan Hospital, Yantai, Shandong 264001, P.R. China
}

Received April 28, 2016; Accepted July 22, 2016

DOI: 10.3892/etm.2016.3539

\begin{abstract}
The aim of the present study was to investigate the characteristics and value of 3D dynamic contrast-enhanced magnetic resonance pulmonary angiography (3D-DCE-MRPA) for the diagnosis of pulmonary embolism (PE). Among patients suspected with PE, 30 cases were scheduled for 3D-DCE-MRPA [magnetic resonance imaging (MRI) group], and 30 cases were examined using multislice computed tomographic pulmonary angiography (msCTPA) [computed tomography (CT) group] Direct signs including location, number, morphology of emboli, and indirect signs such as pulmonary infarction, pneumonia and pleural effusion, were analyzed. Pulmonary artery enhancement was observed. Image quality was contrasted, branches of the pulmonary artery revealed, and differences in sensitivity, specificity and signal-to-noise ratio (SNR) were compared. The number and morphology of emboli in the two groups were compared, and there were no significant differences $(P>0.05)$. In the MRI group, significantly more emboli were located in segmental and subsegmental bronchi $(\mathrm{P}<0.05)$. The indirect signs in the two groups were compared and the differences were not statistically significant $(P>0.05)$. The difference in image quality between the two groups was not statistically significant $(\mathrm{P}>0.05)$. Levels 5 and 6 of the pulmonary artery branch were more evident in the MRI group compared to the CT group. The SNR and carrier-to-noise ratio in the MRI group were significantly higher than those in the $\mathrm{CT}$ group $(\mathrm{P}<0.05)$. Twenty-six cases of $\mathrm{PE}$ were diagnosed in the $\mathrm{CT}$ group, with a sensitivity
\end{abstract}

Correspondence to: Dr Zhongwei Li, CT Room, Yantaishan Hospital, 91 Jiefang Road, Yantai, Shandong 264001, P.R. China E-mail: 1i_zhongwei1@163.com

${ }^{*}$ Contributed equally

Key words: 3D dynamic contrast-enhanced magnetic resonance pulmonary angiography, multislice computed tomographic pulmonary angiography, pulmonary embolism, direct signs, indirect signs, image quality, sensitivity, specificity, signal-to-noise ratio of $90.5 \%$ and specificity of $86.7 \%$. Twenty-five cases were diagnosed in the MRI group, with a sensitivity of $92.3 \%$ and specificity of $84.2 \%$. In conclusion, 3D-DCE-MRPA surpassed msCTPA in revealing segmental and subsegmental pulmonary artery PE.

\section{Introduction}

Pulmonary embolism (PE) is widely acknowledged as an emergency vascular disease (1) that is frequently neglected and misdiagnosed, and can result in mortality. Early symptoms are atypical, while $30-65 \%$ of patients may be asymptomatic. Dyspnea, pectoralgia and hemoptysis are the triad of symptoms of the disease, although they only occur in $3-20 \%$ of patients (2). Acute PE can occur in patients with acute myocardial infarction and aortic dissection (3). Although therapy, including anticoagulation, thrombolysis and thrombectomy, is available, its efficacy are not satisfactory (4).

Lack of awareness in the early diagnosis is an important risk factor (5). The development of diagnostic technologies such as multislice computed tomographic pulmonary angiography (msCTPA) and MRPA have led to improvement in the diagnosis of PE, reaching up to 80-95\% (6). 3D dynamic contrast-enhanced magnetic resonance pulmonary angiography (3D-DCE-MRPA) surpasses msCTPA in screening the pulmonary artery for emboli, reduces X-ray exposure and the negative effects of contrast agent (7).

The aim of the present study was to examine the characteristics of imaging in the diagnosis of PE.

\section{Patients and methods}

Patients. Sixty patients suspected highly of having acute PE were selected between January, 2013 and January, 2016 in the People's Liberation Army 107th Hospital. The exclusion criteria for the study were: Severe cases; $<72 \mathrm{~h}$ of expected survival; complications including acute myocardial infarction, aortic dissection or sudden death; chronic PE; primary lung diseases such as chronic obstructive pulmonary disease, lung cancer or bronchiectasia; failure to end emergent computed tomography (CT) or magnetic resonance imaging (MRI); 
Table I. MRI sequence scanning parameter settings.

\begin{tabular}{|c|c|c|c|c|c|c|c|}
\hline Sequence & TR (msec) & $\mathrm{TE}(\mathrm{msec})$ & TI (msec) & FA (degree) & FOV (cm) & Matrix & Collection time \\
\hline T1WI & 583 & 15 & & $90 / 180$ & $42 \times 42$ & $224 \times 256$ & 2 \\
\hline FSE, T2WI & 3,000 & 100 & & $90 / 160$ & $18 \times 18$ & $192 \times 256$ & 2 \\
\hline 3D FFE & 9 & 3 & 100 & 20 & $40 \times 40$ & $128 \times 256$ & 1 \\
\hline
\end{tabular}

MRI, magnetic resonance imaging; FSE, fast spin echo; FFE, fast field echo.

unqualified image and undiagnosed PE. Thirty cases, including 18 men and 12 women, aged 37-76 years with an average age of $56.4 \pm 13.8$ years, were analyzed using $3 \mathrm{D}$-DCEMRPA. Twenty-two patients had increasing D-dimer levels while 14 had deep venous thrombosis (DVT). Thirty cases, including 17 men and 13 women, aged 39-79 years, with an average age of $57.2 \pm 15.6$ years, were analyzed using msCTPA. Four had increasing D-dimer levels while 12 had DVT.

The study was approved by the Ethics Committee of the the People's Liberation Army 107th Hospital. Informed consent was obtained from the patients and/or their families.

3D-DCE-MRPA. The 3.0T MRI system (MAGNETOM Avanto, Siemens, Erlangen, Germany) and its software INDY R500 Silicon Graphic station were used. The contrast agent used was 469 mg Gd-DTPA (Guangzhou Consun Pharmaceutical Co., Ltd., Guangzhou, China), which was administered intravenously (22GA1.001N, 0.9x25 mm) (Becton-Dickinson, Suzhou, China). Cardiac gating was applied, and standard transverse, coronal and sagittal scans were taken including T1WI, fast spin echo (FSE) and 3D fast field echo (FFE) under the coronal section (Table I). The region of interest (ROI) was found, and a scan slice was set from the pulmonary trunk to the posterior thoracic vertebra. Breath holding was key for the scanning and patients were instructed to hold their breaths for $20 \mathrm{sec}$ at 10 -sec intervals. Four groups of images, including arterial-phase and delayed-phase, were obtained. Maximum intensity projection (MIP) was used for high resolution dimensional reconstructed radiography. Scans were rotated $15^{\circ}$ along their axes, resulting in 13 multi-planar rotated images for observation.

MSCTPA. A Somatom Definition CT scanner was used for examinations from the thoracic inlet to the level of the costophrenic angle. Iopromide (370 mg) was used as the contrast agent, and the scans were taken automatically with the pulmonary trunk as the ROI (threshold, 90-100 HU). The scanning parameters were as follows: Collimation, $0.6 \mathrm{~mm}$; changing pitch, 1.35-1.55 with heart rate; slice thickness, $0.75 \mathrm{~mm}$; reconstructive interval, $0.5 \mathrm{~mm}$; voltage, $120 \mathrm{kV}$; tube current, 180-250 mAs, rotation time, $0.33 \mathrm{sec}$; and scanning time, 4-6 sec. Original data were used for multi-planar reconstruction (MPR), MIP and volume rendering (VR).

Observation indexes. Direct signs including the location, number, and formation of emboli, as well as indirect signs including pulmonary infarction, pneumonia, and pleural effusion, were analyzed. Image quality was contrasted, the pulmonary artery branch was revealed, and differences of sensitivity, specificity and signal-to-noise ratio (SNR) were assessed.

Following embolism, the pulmonary trunk, left and right pulmonary arteries, transverse diameter of the phrenic artery, signal strength of corresponding surface and cervical muscles, and the standard deviation of background noise, were analyzed in the original and MIP reconstructed images. The SNR of corresponding vessels and carrier-to-noise ratio (CNR) between vessels and cervical muscles were calculated as follows: SNR = average signal strength of ROI/noise standard deviation, and $\mathrm{CNR}=\mathrm{SNR}_{\mathrm{vessel}}-\mathrm{SNR}_{\text {cervical muscle }}$. The quality of artery images were divided into four grades. Grade I was poor quality, with blurring of the pulmonary artery segment and part of the subsegment in addition to considerable vein overlap which affected the diagnosis. Grade II appeared normal, with rough segment of the pulmonary artery and part of the subsegment in addition to a moderate amount of pulmonary vein overlap which affected the diagnosis slightly. Grade III images were of good quality, with clearly visible segment of the pulmonary artery part of the subsegment as well as minor vein overlap which conformed to the diagnosis. Grade IV images were optimal, with clear pulmonary artery segment and part of subsegment with little vein overlap which made for accurate diagnosis. The levels of the pulmonary branch were classified as: Pulmonary trunk was level 1, left and right pulmonary arteries were level 2, interlobar artery was level 3, segmental artery was level 4, subsegmental artery (grade I) was level 5, and grade II subsegmental artery was level 6 . The pulmonary artery tapered and any interruption or filling defects that contrasted with its normal formation were considered diagnostic criteria of PE.

Statistical analysis. Data were analyzed using SPSS. Quantitative data are presented as mean \pm standard deviation. Differences among the groups were assessed by t-test and $\chi^{2}$ test. Countable data were expressed as case or percentage (\%). Ranked data were evaluated by Kruskal-Wallis. $\mathrm{P}<0.05$ was considered to indicate a statistically significant difference.

\section{Results}

Comparison of direct signs. The average number and different formations of emboli in the two groups were compared, and there were no significant differences $(\mathrm{P}>0.05)$. There were more emboli appearing in segmental or subsegmental arteries in the MRI group, and the difference was significant $(\mathrm{P}<0.05)$ (Table II). 
Table II. Comparison of direct and indirect signs [cases (\%)].

\begin{tabular}{|c|c|c|c|c|c|c|c|c|c|}
\hline \multirow[b]{2}{*}{ Groups } & \multirow[b]{2}{*}{ Cases } & \multicolumn{4}{|c|}{ Location of embolus } & \multirow[b]{2}{*}{ Numbers } & \multicolumn{3}{|c|}{ Formation } \\
\hline & & $\begin{array}{c}\text { Subsegment } \\
\text { artery }\end{array}$ & $\begin{array}{l}\text { Segmental } \\
\text { artery }\end{array}$ & $\begin{array}{l}\text { Interlobar } \\
\text { artery }\end{array}$ & $\begin{array}{l}\text { Left, right and } \\
\text { trunk of artery }\end{array}$ & & Central & Intraluminal & $\begin{array}{c}\text { Totally } \\
\text { obstructive }\end{array}$ \\
\hline $\mathrm{CT}$ & 30 & $5(16.7)$ & $8(26.7)$ & $16(53.3)$ & $1(3.3)$ & $1.5 \pm 0.2$ & $8(26.7)$ & $15(50.0)$ & $7(23.3)$ \\
\hline MRI & 30 & $10(33.3)$ & $13(43.3)$ & $6(20.0)$ & $1(3.3)$ & $1.6 \pm 0.3$ & $8(26.7)$ & $13(43.3)$ & $9(30.0)$ \\
\hline $\mathrm{t}\left(\chi^{2}\right)$-tes & & \multicolumn{4}{|c|}{$7.024^{\mathrm{a}}$} & 0.627 & \multicolumn{3}{|c|}{0.393} \\
\hline P-value & & \multicolumn{4}{|c|}{0.030} & 0.849 & \multicolumn{3}{|c|}{0.822} \\
\hline
\end{tabular}

${ }^{a}$ Analysis was conducted after data from the interlobar and pulmonary arteries (left, right and trunk) were combined. CT, computed tomography; MRI, magnetic resonance imaging.

Table III. Comparison of indirect signs [cases (\%)].

\begin{tabular}{lccccc}
\hline Groups & Cases & Infarction & Pneumonia & Hydrothorax & Enlargement of pulmonary artery \\
\hline CT & 30 & $4(13.3)$ & $16(53.3)$ & $3(10.0)$ & $7(23.3)$ \\
MRI & 30 & $3(10.0)$ & $15(50.0)$ & $2(6.7)$ & $10(33.3)$ \\
$\chi^{2}$ & & & & 0.909 & 0.823 \\
P-value & & & &
\end{tabular}

CT, computed tomography; MRI, magnetic resonance imaging.

Table IV. Comparison of image quality, pulmonary artery branches and SNR [cases (\%)].

\begin{tabular}{|c|c|c|c|c|c|c|c|c|c|c|}
\hline \multirow[b]{2}{*}{ Groups } & \multirow[b]{2}{*}{ Cases } & \multicolumn{4}{|c|}{ Image quality } & \multicolumn{3}{|c|}{ Branch of pulmonary artery } & \multicolumn{2}{|c|}{ SNR } \\
\hline & & I & II & III & IV & Level 1-3 & Level 4 & Level 5 and 6 & SNR & CNR \\
\hline CT & 30 & $3(10.0)$ & $8(26.7)$ & $13(43.3)$ & $6(20.0)$ & $3(10.0)$ & $20(50.0)$ & $7(23.3)$ & $41.3 \pm 7.6$ & $32.5 \pm 5.9$ \\
\hline MRI & 30 & $2(6.7)$ & $6(20.0)$ & $14(46.7)$ & $8(26.7)$ & $4(13.3)$ & $10(33.3)$ & $16(53.3)$ & $45.6 \pm 8.2$ & $36.7 \pm 6.0$ \\
\hline$t\left(\chi^{2}\right)$-test & & \multicolumn{4}{|c|}{0.812} & \multicolumn{3}{|c|}{7.159} & 5.632 & 5.487 \\
\hline P-value & & \multicolumn{4}{|c|}{0.847} & & 0.028 & & 0.030 & 0.033 \\
\hline
\end{tabular}

SNR, signal-to-noise ratio; CNR, carrier-to-noise ratio; CT, computed tomography; MRI, magnetic resonance imaging.

Comparison of indirect signs. We compared the indirect signs between the two groups, but there were no significant differences $(\mathrm{P}>0.05)$ (Table III).

Comparison of image quality, pulmonary artery branches and SNR. Image quality in the two groups was not statistically different $(\mathrm{P}>0.05)$. There were more level 5 and 6 pulmonary arteries in the MRI group than in the CT group, which also had higher SNR and CNR. The differences were significant $(\mathrm{P}<0.05)$ (Table IV).

Comparison of sensitivity and specificity in diagnosis. Twenty-six cases of PE were confirmed in the CT group, in which sensitivity was $90.5 \%$ and specificity was $86.7 \%$. Twenty-five cases were confirmed in the MRI group, in which sensitivity was $92.3 \%$ and specificity was $84.2 \%$.

\section{Discussion}

The process of imaging by 3D-DCE-MRPA was performed as follows: Paramagnetic contrast agent was injected which shortened the $\mathrm{T} 1$ time to adjacent tissues, including adipose tissue. FFE was selected for scanning of the targeted vessels while patients held their breath. After reconstruction of MIP, well-defined and high-resolution vessel images were obtained. The images were observed at every angle and there was no need to apply spatial pre-saturation, which eliminated disadvantages such as unenhanced MRA, making it suitable for large vessels such as the pulmonary artery. Pulmonary emboli were revealed with filling defects in the pulmonary trunk or branch or with disconnection, blocking, narrowing or deficiency of pulmonary arterial branches (8). It has been shown that if emboli are located in the center of the lumen, a 'track sign' appears (9). 
Compared to msCTPA, 3D-DCE-MRPA allowed for the acquisition of multidimensional images, and the pulmonary vessels in the macropinacoid, middle lobular and lingular artery were seen with precision (10). Therefore, 3D-DCE-MRPA is superior to msCTPA in revealing smaller pulmonary arterial branches. Similarly, examination of the deep vein in the pelvis and lower limbs is convenient and precise (11). PE and deep vein thrombosis are defined as different stages of venous thromboembolism (VTE), advancing the screening of potential PE. Sensitivity in diagnosing PE approached $100 \%$, specificity was $95 \%$, the positive predictive value was $86 \%$ and the negative predictive value was $100 \%$ (12).

Use of msCTPA in diagnosing emboli in the pulmonary trunk, interlobar and segmental artery is highly accurate, with a sensitivity of $94-98 \%$, and specificity of $96-99 \%$. However, diagnosis in the subsegmental and peripheral vessels proved more difficult (13). In addition, more contrast agent was needed for enhanced scanning, which is toxic and can have adverse effects on patients with liver or kidney dysfunction (14). High resolution time leads to monocyclic reconstruction time being shortened to $83 \mathrm{msec}$. This avoids artifacts caused by the heartbeat and breathing and enhances image quality and efficiency of diagnosis. In addition, peripheral vessels, such as the pulmonary artery and aorta were clearly presented (15). Original images, with high spatial resolution provided a high guarantee for postprocessing techniques. The value of isotropic projection reconstruction was obtained, without the misdiagnosis of vertical and subsegmental arteries (16). The requirements for diagnosis and treatment of acute chest pain were met. Standard diagnostic requirements, such as examining for the triad of symptoms of PE, obtaining images of the coronary artery, pulmonary artery and other large vessels in the thorax were unnecessary and were accomplished by a single, rapid scan in order for the diagnoses to be made rapidly and with precision (17). Various post-processing techniques of images guaranteed definite identification of emboli within the pulmonary artery. Intra-arterial filling-defects were shown in MPR. The subtypes were identified and pulmonary artery filling-defects were revealed in the multi-directional and multi-angle phase. Emboli in the horizontal segmental and subsegmental pulmonary arteries were precisely diagnosed (18). The pulmonary artery has been directly visualized with the assistance of MIP and VR technology. In particular, the location of the pulmonary segmental artery has been directly visualized (19), providing exact objective evidence for physicians.

In conclusion, the average number and formations of emboli in the two groups were compared, and no significant differences were observed. There were more emboli appearing in the segmental or subsegmental arteries in the MRI group, and the difference was statistically significant. A comparison of indirect signs between the two groups revealed no significant differences. The image quality in the two groups was not statistically different. Level 5 and 6 pulmonary arteries were observed more in the MRI group than in the CT group, with higher SNR and CNR. The differences were significant. Twenty-six cases of PE were confirmed in the CT group, with sensitivity of $90.5 \%$ and specificity of $86.7 \%$. Twenty-five cases were confirmed in the MRI group, with a sensitivity of
$92.3 \%$ and specificity of $84.2 \%$. Compared with msCTPA, more segmental or subsegmental pulmonary arteries were observed using 3D-DCE-MRPA, with enhanced SNR.

\section{References}

1. Kabrhel C, Rosovsky R, Channick R, Jaff MR, Weinberg I, Sundt T, Dudzinski DM, Rodriguez-Lopez J, Parry BA, Harshbarger S, et al: A multidisciplinary pulmonary embolism response team (PERT) -initial 30-month experience with a novel approach to delivery of care to patients with sub-massive and massive PE. Chest 19: 2-3, 2016.

2. Tan S and Haramati LB: Overdiagnosis versus misdiagnosis of pulmonary embolism. AJR Am J Roentgenol 206: W59, 2016.

3. Rémy-Jardin M, Pistolesi M, Goodman LR, Gefter WB, Gottschalk A, Mayo JR and Sostman HD: Management of suspected acute pulmonary embolism in the era of CT angiography: a statement from the Fleischner Society. Radiology 245: 315-329, 2007.

4. Barco S, Lankeit M, Binder H, Schellong S, Christ M, Beyer-Westendorf J, Duerschmied D, Bauersachs R, Empen K, Held M, et al: Home treatment of patients with low-risk pulmonary embolism with the oral factor Xa inhibitor rivaroxaban. Rationale and design of the HoT-PE Trial. Thromb Haemost 116: 23-24, 2016.

5. van Belle A, Büller HR, Huisman MV, Huisman PM, Kaasjager K, Kamphuisen PW, Kramer MH, Kruip MJ, Kwakkel-van Erp JM, Leebeek FW, et al; Christopher Study Investigators: Effectiveness of managing suspected pulmonary embolism using an algorithm combining clinical probability, D-dimer testing, and computed tomography. JAMA 295: 172-179, 2006.

6. Hutchinson BD, Navin P, Marom EM, Truong MT and Bruzzi JF: Overdiagnosis of pulmonary embolism by pulmonary CT angiography. AJR Am J Roentgenol 205: 271-277, 2015.

7. Yilmaz E, Akkoclu A, Degirmenci B, Cooper RA, Sengun B, Gulcu A, Osma E and Ucan ES: Accuracy and feasibility of dynamic contrast-enhanced 3D MR imaging in the assessment of lung perfusion: comparison with Tc-99 MAA perfusion scintigraphy. Clin Radiol 60: 905-913, 2005

8. Kalb B, Sharma P, Tigges S, Ray GL, Kitajima HD, Costello JR, Chen $\mathrm{Z}$ and Martin DR: MR imaging of pulmonary embolism: diagnostic accuracy of contrast-enhanced 3D MR pulmonary angiography, contrast-enhanced low-flip angle 3D GRE, and nonenhanced free-induction FISP sequences. Radiology 263: 271-278, 2012

9. Ersoy H, Goldhaber SZ, Cai T, Luu T, Rosebrook J, Mulkern R and Rybicki F: Time-resolved MR angiography: a primary screening examination of patients with suspected pulmonary embolism and contraindications to administration of iodinated contrast material. AJR Am J Roentgenol 188: 1246-1254, 2007.

10. Kluge A, Luboldt W and Bachmann G: Acute pulmonary embolism to the subsegmental level: diagnostic accuracy of three MRI techniques compared with 16-MDCT. AJR Am J Roentgenol 187: W7-14, 2006.

11. Stein PD, Chenevert TL, Fowler SE, Goodman LR, Gottschalk A, Hales CA, Hull RD, Jablonski KA, Leeper KV Jr, Naidich DP, et al; PIOPED III (Prospective Investigation of Pulmonary Embolism Diagnosis III) Investigators: Gadolinium-enhanced magnetic resonance angiography for pulmonary embolism: a multicenter prospective study (PIOPED III). Ann Intern Med 152: 434-443, W142-W143, 2010.

12. Keilholz SD, Bozlar U, Fujiwara N, Mata JF, Berr SS, Corot C and Hagspiel KD: MR diagnosis of a pulmonary embolism: comparison of P792 and Gd-DOTA for first-pass perfusion MRI and contrast-enhanced 3D MRA in a rabbit model. Korean $\mathrm{J}$ Radiol 10: 447-454, 2009.

13. Sheh SH, Bellin E, Freeman KD and Haramati LB: Pulmonary embolism diagnosis and mortality with pulmonary CT angiography versus ventilation-perfusion scintigraphy: evidence of overdiagnosis with CT? AJR Am J Roentgenol 198: 1340-1345, 2012

14. Stein PD, Fowler SE, Goodman LR, Gottschalk A, Hales CA, Hull RD, Leeper KV Jr, Popovich J Jr, Quinn DA, Sos TA, et al; PIOPED II Investigators: Multidetector computed tomography for acute pulmonary embolism. N Engl J Med 354: 2317-2327, 2006. 
15. Adams DM, Stevens SM, Woller SC, Evans RS, Lloyd JF, Snow GL, Allen TL, Bledsoe JR, Brown LM, Blagev DP, et al: Adherence to PIOPED II investigators' recommendations for computed tomography pulmonary angiography. Am J Med 126: 36-42, 2013.

16. Ranji SR, Shojania KG, Trowbridge RL and Auerbach AD: Impact of reliance on CT pulmonary angiography on diagnosis of pulmonary embolism: a Bayesian analysis. J Hosp Med 1: 81-87, 2006.

17. Ghanima W, Nielssen BE, Holmen LO, Witwit A, Al-Ashtari A and Sandset PM: Multidetector computed tomography (MDCT) in the diagnosis of pulmonary embolism: interobserver agreement among radiologists with varied levels of experience. Acta Radiol 48: 165-170, 2007.
18. Pena E, Kimpton M, Dennie C, Peterson R, LE Gal G and Carrier M: Difference in interpretation of computed tomography pulmonary angiography diagnosis of subsegmental thrombosis in patients with suspected pulmonary embolism. J Thromb Haemost 10: 496-498, 2012.

19. Costa AF, Basseri H, Sheikh A, Stiell I and Dennie C: The yield of CT pulmonary angiograms to exclude acute pulmonary embolism. Emerg Radiol 21: 133-141, 2014. 